PSICOLOGIA

IBEROAMERICANA
Psicología Iberoamericana ISSN: 1405-0943

revista.psicologia@ibero.mx

Universidad Iberoamericana, Ciudad de México México

\title{
Conducta antisocial adolescente y dinámica familiar. Análisis conceptual
}

Chavarín-García Prieto, María Clara; Gálvez-Hernández, Carmen Lizette

Conducta antisocial adolescente y dinámica familiar. Análisis conceptual

Psicología Iberoamericana, vol. 26, núm. 1, 2018

Universidad Iberoamericana, Ciudad de México, México

Disponible en: http://www.redalyc.org/articulo.oa?id=133959553003 


\title{
Conducta antisocial adolescente y dinámica familiar. Análisis conceptual
}

Adolescent antisocial behavior and family dynamics: A conceptual analysis

\author{
María Clara Chavarín-García Prieto \\ Universidad del Valle de México , México \\ Carmen Lizette Gálvez-Hernández lizettegalvezh@gmail.com \\ Instituto Nacional de Cancerología, México
}

Resumen: La conducta antisocial (ca) en adolescentes ha sido amplio objeto de estudio y preocupación social. Aunque la dinámica familiar y la ca del adolescente tienen una relación bien establecida empíricamente no se tiene una noción integrada de la literatura actual. Objetivo: conocer el estado del arte actual acerca de la influencia de la familia en ca en adolescentes. Método: revisión sistemática en distintas bases de datos a partir de la selección de artículos que cumplan con criterios preestablecidos. Resultados: se obtuvieron 21 artículos. A nivel metodológico, en su mayoría eran longitudinales, que evaluaban tanto a hijos como a padres. Todos mostraron una relación significativa entre las variables, donde el estilo de crianza castigador se encontró con mayor frecuencia relacionado con ca en adolescentes. Conclusiones: la presencia de ca de adolescentes está fuertemente relacionada con la dinámica familiar a través de una interacción bidireccional, influida por distintos niveles de sistemas sociales.

Palabras clave: conducta antisocial, adolescente, familia, dinámica familiar, interacción familiar.

Abstract: Antisocial behavior (ab) in adolescents is a social concern and has been highly studied, due to its numerous social ramifications. Although it is well known that there is a relationship between family dynamics and antisocial behavior amongst adolescents, it is still not fully understood. Objective: To know the current evidence on the effect of family dynamics of the adolescent ab. Method: A systematic review of papers published between 2010 and 2015 in databases was carried out. Results: 21 studies met all the eligibility criteria; they were mainly cross-sectional, descriptive, and correlational studies. All the studies showed a significant relationship between family dynamics and adolescent antisocial behavior. Particularly, authoritarian parenting style was correlated with the antisocial behavior of adolescents. Conclusions: Because children and parents have a significant relationship and bidirectional effect between them, the family with violent or aggressive parenting styles contributes to adolescent antisocial behavior, both influenced by different social systems.

Keywords: antisocial behavior, systematic review, family dynamics, adolescents, family.

\section{INTRODUCCIÓN}

En México cada año 4 mil 500 adolescentes son privados de su libertad por la ejecución de delitos graves (Azaola, 2015). En el 2014, se registraron 16 mil 885 casos de adolescentes que recibieron sanciones por haber cometido algún tipo de infracción. De ellos, 4 mil 558 (27\%) fueron privados de su libertad.

Según el Instituto Nacional de Estadística y Geografía (inegi), en el 2012, en México, se registraron 44 mil 852 conductas antisociales (ca) llevadas a cabo por adolescentes. Estas conductas tienen un impacto 
directo dentro del gasto social a través de los procesos judiciales relacionados con la procuración de justicia, investigación, intervención y rehabilitación. En este sentido, un meta-análisis (Gabor, 2016) que identificó 65 estudios con datos originales sobre los costos del crimen, provenientes de Estados Unidos, Canadá, Australia y Reino Unido, concluyó que éstos (en dólares americanos) varían enormemente por delito y por tipo de procuración de justicia criminal. A pesar de ello, en general, se calcula que la custodia juvenil cuesta 51 mil 742 dólares por cada joven; la custodia condicional (o abierta), alrededor de 926 dólares por joven. Los procesos de la corte se cotizan entre mil 445 y 44 mil 280 dólares, dependiendo de la unidad de análisis (por ejemplo, por caso o por condena). Finalmente, se estima que los arrestos costaron 15 mil 364 dólares. Incluso, Gabor (2016) enfatiza que el costo del crimen varía por país, edad y método de estimación de éstos.

Las ca se han definido como comportamientos dirigidos contra normas, valores y principios de la comunidad a la que el individuo pertenece, incluyendo todos los tipos de conductas de alto riesgo (Bonino, Cattelino, \& Ciairano, 2005). Gaeta y Galvanovski (2011) agregan que las ca hacen referencia a comportamientos agresivos e impulsivos, así como a trastornos o problemas de la conducta, entre otros. Todo ello puede llevar consigo un riesgo directo sobre la salud física y psicológica, tanto de los adolescentes (y su contexto social próximo), como sobre la propiedad y calidad de vida de las víctimas (Ibabe \& Jaureguizar, 2013). Sin embargo, no existe consenso sobre aquellos comportamientos, como elementos conceptuales, que definen las ca. Si bien no todas las ca se encuentran tipificadas como delito, en la mayoría de los casos anteceden el inicio de conductas delictivas (Alvarado \& Cruz, 2004). De hecho, Moffitt et al. (2006) lograron identificar que gran parte de los adultos que presentaron ca tenían antecedentes de problemas de conducta en la infancia y ca en la adolescencia.

Diversos estudios han confirmado que situaciones de la dinámica familiar, estructura familiar, integrantes, nivel económico, estrés derivado de la situación económica, cultura, así como las prácticas de crianza (por ejemplo, el monitoreo y el control parental), son un factor influyente de estas conductas (Blatný, Hrdlička, Jelínek, \& Sobotková, 2012; Bolland, Spano, \& Vazsonyi, 2009; Evans, Gordon, \& Simons, 2016; Moffitt et al., 2006; Ochieng, 2014). Específicamente, el papel del conflicto y la violencia familiar se ha señalado como favorecedor de la transmisión de ca en los adolescentes (Ehrensaft \& Cohen, 2011). Inclusive, se destaca el impacto de las pautas de crianza y el vínculo padre-hijo en el desarrollo psicoemocional del sujeto (Collishaw, Gardner, Maughan, Scott, \& Pickles, 2012).

Por tanto, de acuerdo con las consecuencias sociales, psicológicas y económicas, se resalta la importancia de tener un conocimiento actualizado de los hallazgos sobre el papel de la familia en la presencia de ca en adolescentes, de manera que se obtenga una comprensión más integrada (metodológica y conceptual) del fenómeno, que permita 
revisar marcos conceptuales, así como el replanteamiento de las metas de intervención y rehabilitación de estas conductas.

Por lo que el objetivo general del presente estudio es determinar la influencia de la familia en las ca de adolescentes a través de una revisión sistemática entre los años 2010 y 2015. Los objetivos específicos son: a) describir las características metodológicas; b) identificar aportaciones teóricas de la literatura; y c) identificar los componentes conceptuales de la definición de ca.

\section{MÉTODO}

Para alcanzar los objetivos se realizó una revisión sistemática de la literatura de interés, a través del siguiente proceso de selección.

\section{Criterios de selección}

Se incluyeron artículos de tipo empírico, cuantitativo, cualitativo, longitudinal y transversal, publicados entre los años 2010 y 2015 en bases de datos académicas. La edad máxima de la muestra fue de hasta 17 años; además de que entre sus objetivos de estudio se explorara la participación directa de la familia.

\section{Proceso de selección}

En las bases de datos EBSCO, ProQuest y Ovid se identificaron artículos sólo en el idioma inglés a través del uso de las palabras clave: "adolescent", "antisocial behavior", "family", "teenagers", "parenting style", "family structure". A través de la lectura del resumen de los artículos encontrados, se excluyeron los que por su naturaleza y objetivo de investigación no cumplían con los criterios de inclusión. Véase Figura 1, para mayor detalle.

\section{Análisis de datos}

El análisis de la información se basó en a) definición de ca; b) objetivo y diseño del artículo; c) uso de instrumentos de medición; y d) población a evaluar (padres, hijos adolescentes o ambos). Con respecto a los adolescentes, la información se organizó en término de edad y nivel educativo (obteniendo el porcentaje correspondiente con base en la cantidad de artículos). En el caso de los padres, se consideró nivel educativo, tipo de familia (con base en los integrantes y la relación consanguínea entre ellos), ascendencia y nivel socioeconómico (incluyendo los ingresos económicos). Las aportaciones a la literatura se localizaron en los resultados estadísticos significativos entre dinámica familiar y la presencia de las ca en adolescentes. Los componentes conceptuales de las ca se identificaron a través de las conductas descritas, su definición, así como la frecuencia con la que estos elementos coincidieron. 


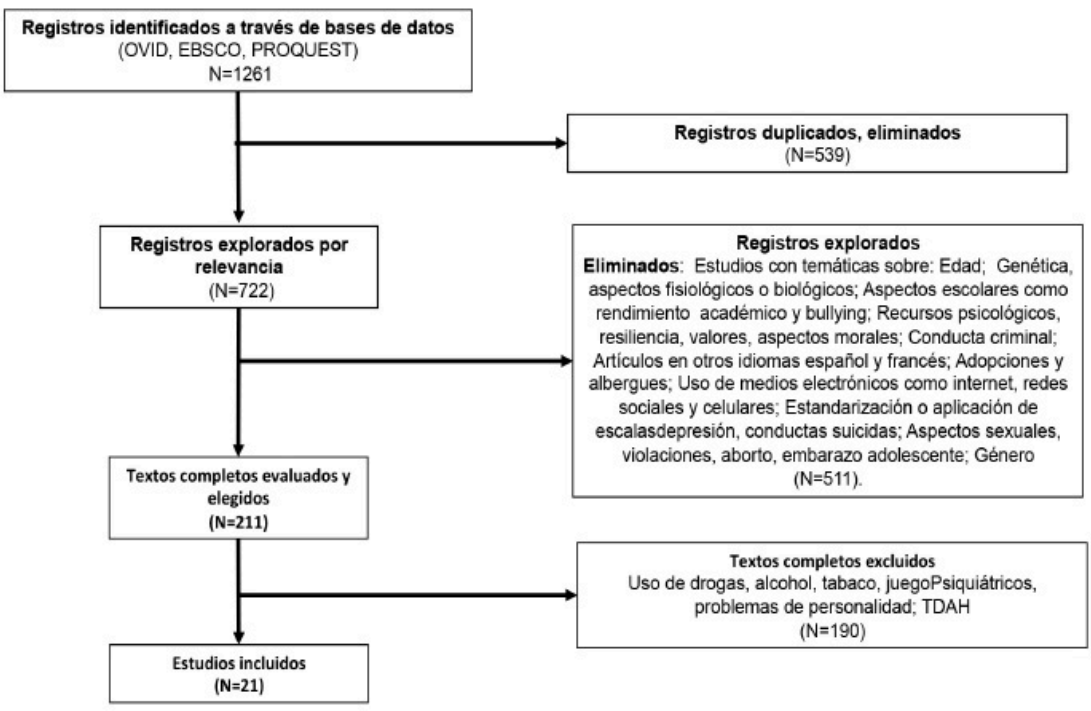

Figura 1

Proceso de selección y clasificación de artículos identificados

Figura 1

Proceso de selección y clasificación de artículos identificados

\section{RESULTADOS}

De 928 artículos que se hallaron en las bases de datos, sólo 21 cumplieron con los criterios establecidos (realizados entre 2010 y 2015 , artículos empíricos, etcétera) en muestras adolescentes entre 9 y 17 años, que evaluaron la participación directa de la familia en el desarrollo, mantenimiento, impulso o como agente de las ca en adolescentes. Véase a detalle en la Figura 1.

De los artículos que fueron descartados llama la atención que gran parte de éstos estudió la asociación entre las ca y problemas de tipo psiquiátrico y biológico, conducta criminal y vandalismo, aspectos relacionados con la escuela (bullying), uso de redes sociales, la aplicación de pruebas psicológicas, intervención psicológica, entre otros.

\section{DESCRIPCIÓN DE LAS CARACTERÍSTICAS METODOLÓGICAS}

\section{Por objetivo}

Los estudios coincidieron en evaluar la relación entre las ca en adolescentes y los estilos educativos de los padres a través de términos como a) prácticas de gestión familiar para el control conductual de los adolescentes; b) prácticas parentales, referidas en el estilo de crianza, como autoritario, permisivo, indiferente, castigador o democrático; c) parentalidad deficiente, por ejemplo, al utilizar la fuerza física como coerción o abandono emocional de los padres; d) factores familiares, como 
la composición de la familia, nivel educativo y edad de los padres; e) dinámica familiar, como el tiempo de convivencia entre los padres e hijos, presencia continua o ausencia de los padres; f) monitoreo, refiriéndose al control no agresivo sobre los adolescentes al tener conocimiento sobre sus actividades y amistades; y, g) factores ambientales, tales como nivel socioeconómico de la familia y vulnerabilidad de la zona de residencia de los adolescentes. De éstos, $28.57 \%$ aborda como objetivo la parentalidad deficiente y agresiva; $23.80 \%$, el monitoreo parental y prácticas parentales; mientras que $19.04 \%$, la dinámica familiar y factores ambientales.

\section{Por diseño metodológico}

$38.09 \%$ fue de tipo transversal, mientras que $61.90 \%$, de tipo longitudinal. De éstos, 38.09\% tuvo un alcance correlacional; $28.57 \%$, predictivo; $y$, $33.33 \%$ descriptivo.

\section{Instrumentos de medición}

47.61\% de los estudios aplicaron instrumentos a padres e hijos: $38.09 \%$ evaluó exclusivamente a los menores. Dichos instrumentos consistieron en auto-reportes o escalas que evaluaron, entre otros constructos, la salud social, ca y criminal, comportamientos hacia la autoridad, interacción padre-hijo, conducta en niños, ambiente y funcionamiento familiar.

\section{Población evaluada}

Los estudios evaluaron diversos rangos de población. Algunos consideraron familias, en promedio $\mathrm{N}=350$ familias por artículo; y otros sólo evaluaron a adolescentes, en promedio $\mathrm{N}=800$ por artículo. De los estudios que registraron el nivel educativo de los adolescentes, 9.52\% tenían nivel bachillerato y $42.85 \%$ nivel secundario; $47.61 \%$ de los artículos no lo consideró relevante. Respecto al tipo de familia, los estudios encontraron que $28.57 \%$ vivía en una familia mono-parental, $4.76 \%$ en familias reconstituidas, $4.76 \%$ con familias biparentales y 23.80\% más de dos tipos de familia; $23.80 \%$ no especificaron el tipo de familia de los adolescentes. En cuanto al nivel académico de los padres, se encontró que $42.85 \%$ de los estudios seña- laban que los padres tenían nivel secundario, y $9.52 \%$ nivel bachillerato; $57.14 \%$ no lo consideraron. Con respecto al ingreso económico de la familia, $23.80 \%$ de los artículos identificó a las familias de bajos recursos (utilizando adjetivos como low income, below the poverty line, high risk), mientras que $76.19 \%$ no hicieron énfasis en esta variable.

\section{Aportaciones teóricas a la literatura}

El estilo parental castigador fue el que más frecuentemente (en $23.80 \%$ de los estudios) se relacionó positivamente con la presencia de ca en 
adolescentes; seguido del estilo indiferente (14.28\%), autoritario (9.52\%), la combinación del estilo indiferente y castigador (9.52\%) y, finalmente, el estilo permisivo (4.76\%). De acuerdo con este análisis se identificaron los siguientes datos relevantes, para ampliar la comprensión del tema: a) los varones fueron más agresivos que las mujeres, utilizando en mayor medida la fuerza física, como mecanismo de coerción; b) la presencia de violencia familiar se percibió como un generador de ca, no así el nivel socio-económico ni la estructura familiar; c) la ca en adolescentes incrementa con la edad y, si se presentó en la adolescencia temprana, se reproducirá en la adolescencia tardía; d) la impulsividad y enojo por parte de los adolescentes correlacionó positivamente con la presencia de ca, así como negativamente con la percepción de recibir menos atención, implementación de límites ambiguos e indefinidos y menor demostración de afecto. Véase a detalle Tabla 1.

Por el contrario, los estilos de crianza que se relacionaron negativamente con la presencia de ca fueron: el democrático (14.28\%) y monitoreo (14.28\%); 9.52\% de los estudios identificaron la combinación de éstos. En este sentido, se encontró también que el uso de técnicas de monitoreo, control y comunicación adecuadas, por un lado, favoreció la disminución de ca en adolescentes; y por el otro, generó una relación cercana con los padres, permitiendo que los adolescentes aceptaran el monitoreo y control. Se observó que la familia percibida por los adolescentes como generadora de redes de apoyo y de orientación actuó como factor protector frente a la presencia de ca. Además, se identificó que las conductas prosociales y las habilidades en la resolución de conflictos correlacionaron negativamente con el consumo de drogas y ca.

\section{Componentes conceptuales de la ca}

El 17.94\% de los artículos incluyeron una definición sobre ca. De estas definiciones, los elementos que consideraron como parte de éstas, con más frecuencia, fueron: a) consumo de sustancias (alcohol y drogas); b) daño a otras personas dentro del plano físico, mental o en su patrimonio; $y, c$ ) acciones que rompen reglas, respectivamente.

\section{DISCUSIÓN}

El objetivo de esta investigación consistió en determinar la influencia de la familia en las ca de adolescentes en función del análisis de investigaciones recientes.

Todos los artículos demostraron la existencia de una relación significativa entre ca de adolescentes y dinámica familiar. Es posible que este resultado dé cuenta de la importancia de la familia y de las interacciones que suceden dentro de ella, ya que ésta se ha considerado la matriz psicosocial en donde el sujeto aprenderá a relacionarse con la sociedad; favoreciendo la repetición de la educación familiar, y siendo permeada por el contexto cultural y social (Macías, 2012). A la par, esta 
importancia se explica porque es el ambiente social donde los padres aparecen como figuras de formación, que ejercen un modelado sobre los hijos (Giannotti, Nardone, \& Rocchi, 2008).

La correlación entre ca en adolescentes y estilo castigador se comprende a través de que el ejercicio de una parentalidad hostil genera deficiencias emocionales en los hijos que conllevan al desarrollo de este tipo de conductas (Carrasco, Holgado, Rodríguez, \& Del Barrio, 2009). La conducta conflictiva de los adolescentes se deduce relacionada al uso de técnicas poco asertivas por parte de los padres como mecanismos de control; tales como castigos intermitentes, agresiones físicas y abandono emocional hacia los adolescentes (Hoeve et al., 2009).

$\mathrm{Al}$ parecer, el estilo castigador envía la información a los hijos sobre la facilidad para el rompimiento de reglas y la devaluación de las figuras de autoridad; y, dado que los padres son el primer modelo para la adquisición de conductas socialmente aceptadas, los adolescentes pueden trasladar este tipo de conductas a otras figuras de autoridad, impactando dentro del sistema social (Suárez \& Menkes, 2006).

Esta relación entre conducta de padres e hijos ha sido descrita por Arranz (2004) como un proceso interaccional-bidireccionaltransaccional-dialéctico, ya que la comunicación corre en los dos puntos de manera continua, lo que conlleva una influencia directa entre las respuestas de los hijos y las de los padres, de tal manera que se retroalimentan mutuamente. La comunicación se mantiene a través de dos subsistemas familiares (padres e hijos), lo que le da el carácter de transaccional; y, al ser ininterrumpido a lo largo de la historia de vida de los padres, la relación con sus hijos adquiere un sentido dialéctico (Oliva, 2006).

Según Arranz (2004), estas conductas también se encuentran influenciadas a través de la interacción con sistemas más globales (mesosistema, exosistema y macrosistema), es decir, a través de la interacción de la red mesosistémica que incluye a los amigos, vecinos e iguales que se comprometen en conductas similares. Del exosistema que implica la familia extensa como los abuelos, tíos (as), primos (as), etcétera. Por último, influye de manera indirecta el macrosistema en el cual se insertan aspectos socio-culturales, tales como la estructura socioeconómica marginada o de bajos recursos, etcétera, donde posiblemente se perciben de manera positiva las ca (Gálvez-Hernández, 2006; Silva, 2003; Wilson \& Herrnstein, 1985).

Metodológicamente, la mayoría de los estudios fueron de tipo longitudinal lo que da cuenta de la eficiencia, robustez y potencia de los estudios (Jaume \& Roser, 2008). Igualmente, la mayoría evaluó a padres e hijos, lo que puede estar dando cuenta de que los estudios suponen un modelo familiar donde se debe capturar la percepción de los dos miembros de la díada.

Al parecer, el perfil más común de familias que caracterizan a estos adolescentes son monoparentales y constituidas. Según Tamez y Ribeiro (2016) el incremento de familias monoparentales se encuentra asociado al aumento de divorcios y a la transformación social y demográfica, 
caracterizada por el aumento de la escolaridad de la población, la incorporación de la mujer al mercado laboral, la centralidad de la vida familiar en la pareja, el mayor valor y atención hacia los hijos, mayor tiempo de dependencia de éstos al núcleo familiar y el incremento de la esperanza de vida.

La ausencia de relación entre variables educativas/ económicas y las ca como hallazgo puede desmitificar el hecho de que los sistemas familiares con menos recursos económicos son favorecedores de la presencia de ca en adolescentes, con lo cual se enfatiza la importancia de las habilidades familiares para ejecutar pautas de crianza más adecuadas, en contraste con características que pudieran estar en menor control de la familia. Por tanto, aunque los elementos socioeconómicos pueden influir en la tarea de criar, pareciera que si los padres cuentan con las herramientas y habilidades psicológicas suficientes para desarrollar pautas de crianza asertivas, podrán favorecer la resiliencia de sus hijos (Rodrigo \& Martín, 2009).

\section{CONCLUSIONES}

Las ca en adolescentes son un problema social de gran importancia en todos los niveles implicados. Para su comprensión, la influencia familiar ha sido un tema frecuentemente estudiado. Como se ha mostrado, los padres dentro del sistema familiar son relevantes en lo que al comportamiento de los hijos se refiere. La forma en cómo los primeros se relacionen con los hijos a través de su crianza puede favorecer o no la presencia de ca, ya que padres que ejecutan estilos de crianza castigador, indiferente, autoritario o permisivo aumentan la posibilidad de ejecución de este tipo de conductas, a partir de la violencia o el abandono ejercido sobre los menores. Por el contrario, los estilos de crianza democrático y monitoreo de los hijos disminuyen la posibilidad de ejecución de ca. Aunque estos resultados asumen a los padres como los únicos responsables del comportamiento de los hijos, en este artículo se revisa un marco conceptual (Arranz, 2004; Wilson \& Herrnstein, 1985) que propone que el comportamiento del hijo (y del padre) es producto de una relación bidireccional, transaccional y dialéctica; aunado al efecto de los diferentes niveles de sistemas sociales. Por lo que se plantea que las ca son producto de una influencia social compleja y dinámica a diferentes niveles de interacción.

Metodológicamente se observaron dos puntos a destacar de la literatura revisada. En primer lugar, los estudios carecen de diseños con grupos de comparación y diseños de intervención, lo que no permite alcanzar un mayor nivel de comprensión y atención del fenómeno. En segundo lugar, la mayoría de éstos se concentró en revisar las características negativas de la interacción familiar a través de los estilos educativos y, en menor medida, los factores globales como la dinámica familiar, los estilos de crianza favorecedores de factores de protección y generadores de conductas prosociales; lo cual puede implicar un sesgo, ya que las problemáticas no sólo pueden manejarse a través de su conocimiento e investigación, sino 
también desde el fortalecimiento epistémico de la contraparte teórica, lo cual es factor de cambio y reto académico. La ausencia de consenso sobre la definición de ca puede estar contribuyendo a la falta de integración de los resultados de las diversas investigaciones en el área, lo que a su vez no ha permitido obtener un conocimiento que dé respuesta sólida a la problemática; y explicar el alcance inconsistente encontrado en los resultados de intervención, dirigidas a las ca en adolescentes. Por lo que en estudios futuros sería útil identificar elementos precisos y estandarizados que permitan aproximarse directa y consensada al fenómeno de interés.

Paralelamente, podemos notar que al nivel de cada país existen dos barreras importantes que pueden estar dificultando el avance del estudio del fenónomeno (principalmente en países en desarrollo), en primer lugar, la carencia de datos epidemiológicos a nivel nacional actualizados y homogéneos, así como la falta de instrumentos de evaluación adaptados culturalmente a las muestras de interés.

La limitación de este estudio puede encontrarse en las dificultades que se tuvieron para acceder a las bases de datos, generando conclusiones reducidas o sesgadas. Empero, asumir el modelo teórico Ecológico y el Transaccional-Bidireccional (Arranz, 2004) para la interpretación de los resultados de este estudio, parece haber sido una herramienta conceptual adecuada, ya que permitió alcanzar una visión más integral y exhaustiva de los mecanismos de influencia familiar en la ca del adolescente. Lo que, a su vez, proporciona nuevas alternativas de abordaje metodológico, teórico y social de este fenómeno, que representa un gran desafío por los costos sociales, personales y familiares. 


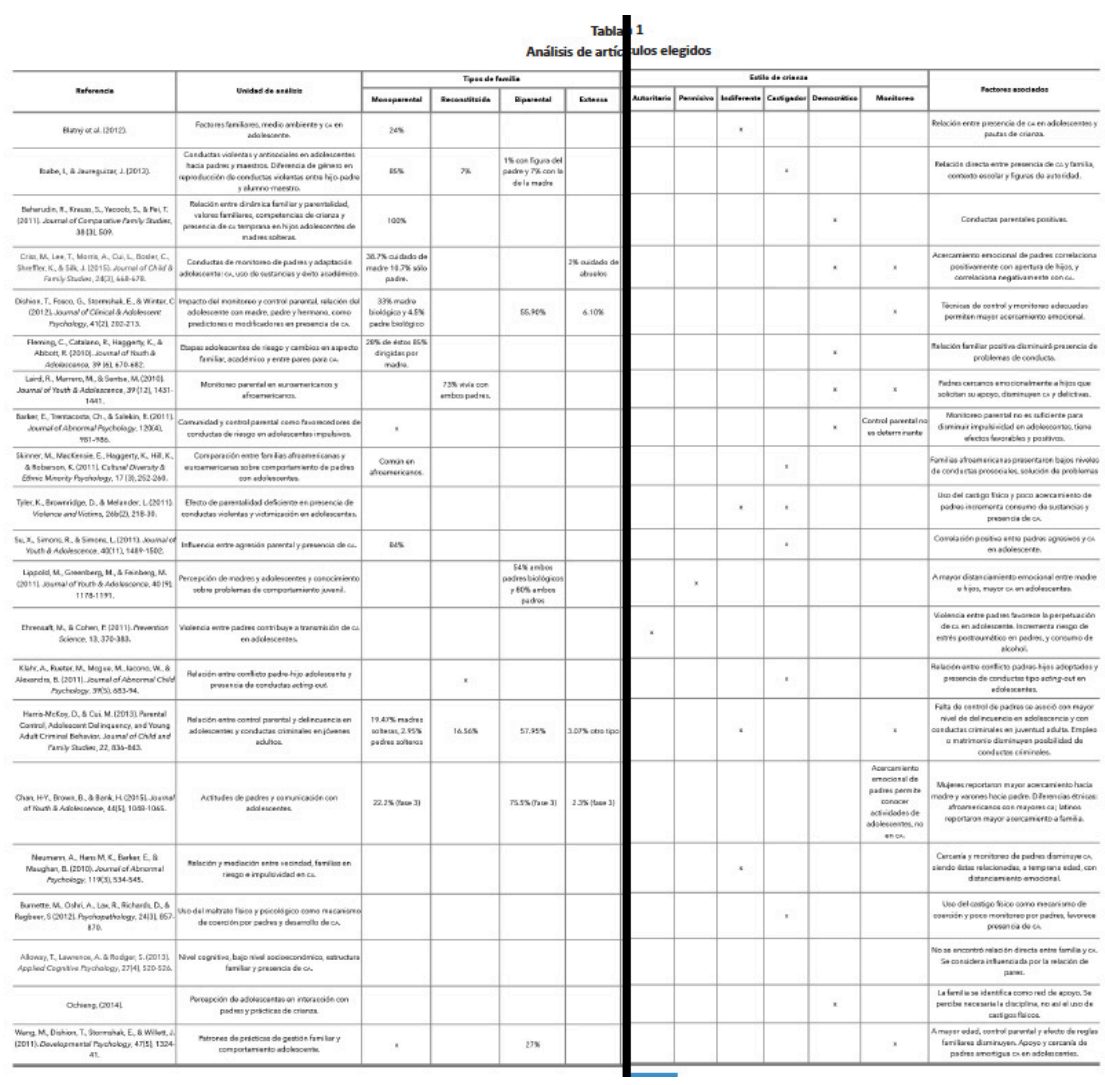

Tabla 1

Análisis de artículos elegidos

\section{REFERENCIAS}

Alvarado, A., \& Cruz, A. (2004). La conducta antisocial en adolescentes, su relación con el entorno familiar y la percepción de riesgo social, una base para la prevención de conductas delictivas (Tesis de licenciatura). Universidad Nacional Autónoma de México, Ciudad de México.

Arranz, E. (2004). Familia y desarrollo psicológico. México: Pearson Educacion.

Azaola, E. (2015). Diagnóstico de las y los adolescentes que cometen delitos graves en México. México: Unicef

Blatný, M., Hrdlička, M., Jelínek, M., \& Sobotková, V. (2012). Antisocial behavior in adolescence: Typology and relation to family context. Journal of Early Adolescence, 33(8), 1091-1115. https://doi.org/10.1177/027243 1612445377

Bonino, S., Cattelino, E., \& Ciairano, S. (2005). Adolescents and risk. Behaviors, functions and protective factors. New York, NY, US: Springer.

Bolland, J., Spano, R., \& Vazsonyi, A. (2009). Does parenting mediate the effects of exposure to violence on violent behavior? An ecological-transactional model of community violence. Journal of Adolescence, 32(5), 1321- 1341. https://doi.org/10.1016/j.adolescence.2008.12.003

Carrasco, M., Holgado, F., Rodríguez, M., \& Del Barrio, M. (2009). Concurrent and across-time relations between mother/father hostility and children's aggression: A longitudinal study. Journal of Family Violence, 24(4), 213-220. https://doi.org/10.1007/s10896-009-9222-y 
Collishaw, S., Gardner, F., Maughan, B., Scott, J., \& Pickles, A. (2012). Do historical changes in parent-child relationships explain increases in youth conduct problems? Journal of Abnormal Child Psychology, 40(1), 119-132. https://doi.org/10.1007/s10802-011-9543-1

Ehrensaft, M., \& Cohen, P. (2011). Contribution of family violence to the intergenerational transmission of externalizing behavior. Prevention Science, 13(4), 370-383. https://doi.org/10.1007/s11121-011-0223-8

Evans, S., Gordon, L., \& Simons, R. (2016). Factor that influence trajectories of delinquency throughout adolescence. Journal of Youth Adolescence, 45(1), 156-171. https://doi.org/10.1007/s10964-014-0197-5

Gabor, T. (2016). Cost of crime and criminal justice responses: Research report 2015-R022. Public Safety Canada. Recuperado de www.publicsafety.gc.ca

Gaeta, M., \& Galvanovskis, A. (2011). Propensión a conductas antisociales y delictivas en adolescentes mexicanos. Psicología Iberoamericana, 19(2), 47-54.

Gálvez-Hernández, C. (2006). Tasas de descuento: Un estudio exploratorio en menores infractores (Tesis de licenciatura). Universidad Nacional Autónoma de México, Ciudad de México.

Giannotti, E., Nardone, G., \& Rocchi, R. (2008). Modelos de familia. España: Herder.

Hoeve, M., Semon, J., Eichlsheim, V., Van der Laan, P., Smeenk, W., \& Gerris J. (2009). The relationship between parenting and delinquency: A metaanalysis. Journal of Abnormal Child Psychology, 37(6), 749- 775. https:// doi.org/10.1007/s10802-009-9310-8

Ibabe, I., \& Jaureguizar, J. (2013). Violent and prosocial behavior by adolescents toward parents and teachers in a community sample. Psychology in the Schools, 50(5), 451-470. https://doi.org/10.1002/pits.21685

Instituto Nacional de Estadística y Geografía (inegi) (2012). Censo nacional de procuración de justicia estatal. Recuperado de http:// www.beta.inegi.org.mx/proyectos/censosgobierno/estatal/cnpje/2012/? init $=2$.

Jaume, A., \& Roser, B. (2008). Estudios longitudinales: Modelos de diseño y análisis. Escritos de Psicología-Psychological Writings, 2(1), 32-41.

Macías, R. (2012). Entendiendo y tratando el corazón de la familia. México: El Saber Instituto.

Moffitt, T. E., Trzesniewski, K. H., Donnellan, M. B., Robins, R. W., Poulton, R., \& Caspi, A. (2006). Low self-esteem during adolescence predicts poor health, criminal behavior, and limited economic prospects during adulthood. Developmental Psychology, 42(2), 381-390. https://doi.org/10 $.1037 / 0012-1649.42 .2 .381$

Ochieng, B. (2014). Minority ethnic adolescent's well-being: Child rearing practices and positive family influences. Health Education Journal, 73(3), 324-331. https://doi.org/10.1177/0017896912471053

Oliva, A. (2006). Relaciones familiares y desarrollo adolescente. Anuario de Psicología, 37(3), 209-223.

Rodrigo, M., \& Martín, J.C. (2009). Las competencias parentales en contextos de riesgo psicosocial. Psychosocial Intervention, 18(2), 113-120.

Silva, A. (2003). Criminología y conducta antisocial. México: Pax. 
Suárez, L., \& Menkes, C. (2006). Violencia familiar ejercida en contra de los adolescentes mexicanos. Revista de Saúde Publica, 40(4), 611-619.

Tamez, B., \& Ribeiro, M. (2016). El divorcio, indicador de transformación social y familiar con impacto diferencial entre los sexos: Estudio realizado en Nuevo León. Papeles de Población, 22(90), 229-263. http://dx.doi.org/10 $.22185 / 24487147.2016 .90 .040$

Wilson, J., \& Herrnstein, R. (1985). Crime and human nature. New York, NY, US: Shine \& Shuster. 